\title{
Massive Anti-de Sitter gravity from string theory
}

\section{Constantin Bachas and loannis Lavdas}

Laboratoire de Physique Theorique de l'Ecole Normale Superiéure, PSL University, CNRS \& Sorbonne Universités 24 rue Lhomond, 75231 Paris Cedex, France

E-mail: bachas@lpt.ens.fr, lavdas@lpt.ens.fr

AbSTRaCt: We study top-down embeddings of massive Anti-de Sitter (AdS) gravity in type-IIB string theory. The supergravity solutions have a $\mathrm{AdS}_{4}$ fiber warped over a manifold $M_{6}$ whose shape resembles that of scottish bagpipes: the 'bag' is a conventional $\mathrm{AdS}_{4}$ compactification manifold, while the 'pipes' are highly-curved semi-infinite Janus throats. Besides streamlining previous discussions of the problem, our main new result is a formula for the graviton mass which only depends on the effective gravitational coupling of the bag, and on the D3-brane charges and dilaton jumps of the Janus throats. We compare these embeddings to the Karch-Randall model and to other bottom-up proposals for massive AdS gravity, and we comment on their holographic interpretation. This is a companion paper to [1], where some closely-related bimetric models with pure $\mathrm{AdS}_{5} \times \mathrm{S}^{5}$ throats were analyzed.

KEYwords: AdS-CFT Correspondence, Flux compactifications

ARXiv EPrint: 1807.00591 


\section{Contents}

1 Introduction $\quad 1$

2 Mass as holographic leakage 3

3 Recombination of representations 4

4 Scottish bagpipes $\quad 5$

5 Mass from Janus throats $\quad 8$

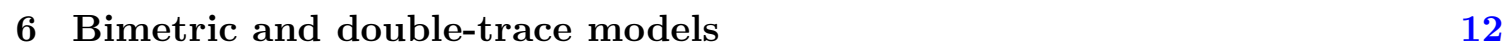

$\begin{array}{lll}7 & \text { Concluding remarks } & 14\end{array}$

$\begin{array}{ll}\text { A The type IIB solutions } & 15\end{array}$

\section{Introduction}

Efforts to endow the graviton with a tiny mass have a long history, going back to the work of Fierz and Pauli [2] and continuing unabated today - for reviews and references see [35]. The problem is of obvious theoretical interest, and could have far-reaching implications for cosmology. In one recent development it has been argued that a key obstruction to the graviton mass - the appearance of a Boulware-Deser ghost [6], can be removed in certain classical non-linear extensions of the Fierz-Pauli action [7-9] (see also [10, 11]). Questions, however, remain both in what concerns the consistency of such classical theories, and with regards to their range of validity if viewed as effective field theories around a given classical background. ${ }^{1}$

One may hope to answer such questions by embedding massive gravity in an ultravioletcomplete theory like string theory. In this paper we will consider four-dimensional Anti-de Sitter (AdS) solutions of IIB string theory in which the lowest spin-2 mode has a tiny mass $m_{\mathrm{g}}$. In discussions of massive gravity, AdS is known to be an 'easier case' since it does not suffer from some of the difficulties encountered in the Mikowski and de Sitter backgrounds. There is, in particular, no van Dam-Veltman-Zakharov discontinuity [19, 20], and hence no need for the strong non-linearities known as Vainshtein screening [21]. It remains therefore to be seen whether our embeddings of massive AdS gravity carry any lessons for these other backgrounds.

\footnotetext{
${ }^{1}$ For a very partial list of references raising or trying to address such questions see [12]-[18] and the above reviews.
} 
The general idea behind the embeddings, due to Karch and Randall [22, 23], is to 'locally localize' the graviton on an $\mathrm{AdS}_{4}$ brane living in $\mathrm{AdS}_{5}$ bulk. Using a thin-brane approximation these authors showed that if the ratio of AdS radii is small, $L_{5} / L_{4} \ll 1$, the lightest graviton mode acquires a tiny mass. The proper string-theory realization of the idea had to wait for the derivation of exact solutions describing intersecting D3, D5 and NS5-branes [24]-[29]. One key departure from the Karch-Randall model is the failure of the thin-brane approximation for the localizing brane, which is a D3-D5-NS5 bound state. As shown in [26] the AdS radius of this composite brane cannot be made parametrically larger than its thickness. As a result the Kaluza-Klein scale (beyond which any $4 d$ description must break down) is $L_{4}$, and not $L_{5}$ as in ref. [23]. This is related to the familiar scale separation problem of AdS flux vacua, for a discussion see [30-32]. ${ }^{2}$

The purpose of the present note is to derive an (almost universal) formula for the graviton mass in these string-theory embeddings. This is a follow-up paper to ref. [1] which analyzed closely related embeddings of bigravity models. Apart from the change in emphasis compared to [1], we will here also extend the results of this reference by allowing the dilaton to vary in the $\mathrm{AdS}_{5}$ bulk which is deformed to the supersymmetric Janus background [24]. This modifies the graviton mass by a multiplicative factor that we will compute. Our formula for the graviton mass is derived on the gravity side. It is an interesting open problem to match it with a computation of the anomalous dimension of the energy-momentum tensor on the CFT side.

There have been two other proposals in the literature for realizing massive AdS gravity in string theory. They relied either on transparent boundary conditions in AdS [33-35], or on multi-trace deformations in CFT [36-38]. In these proposals the graviton mass is a quantum, one-loop effect. Although our embedding could be possibly rephrased in these other frameworks by integrating out messenger degrees of freedom, it has the advantage of relying on proper classical solutions of $10 d$ supergravity. It does not therefore suffer from some difficulties of the above proposals, namely non-locality of the worldsheet theory, or hard-to-control renormalization group flows [39-41]. The 'price to pay' is that the graviton mass is quantized and cannot be tuned continuously to zero. We will return to this point towards the end of the paper.

The paper is organized as follows: in section 2 we recall why defect or interface CFT [43] - [46] is the appropriate holographic setup for Higgsing the AdS graviton. Holographic duality is not crucial to our later analysis, but it provides useful insights on the underlying mechanism. Section 3 explains the group theory of the Higgsing, i.e. the recombination of representations of the $\mathcal{N}=4$ superconformal algebra $\mathfrak{o s p}(4 \mid 4)$ which is the symmetry of the relevant background solutions. This section can be skipped without affecting the flow of the paper.

Section 4 describes the qualitative characteristics of the supergravity solutions that lead to a small graviton mass. These solutions consist of $\mathrm{AdS}_{4}$ fibers warped over sixdimensional manifolds with the shape of scottish bagpipes. The 'bag' describes a standard

\footnotetext{
${ }^{2}$ As explained in ref. [30] scale non-separation is unavoidable for solutions with a continuous R-symmetry, i.e. for the vast majority of known solutions of $10 d$ supergravity including the ones discussed here. The authors of this reference outline possible ways out of the problem.
} 
$\mathrm{AdS}_{4}$ compactification, while the non-compact 'pipes' are highly-curved Janus throats. In section 5 we calculate, following [1], the graviton mass to leading order in the throat-to-bag size, and show that it only depends on few parameters of the solutions: the radius and dilaton variation in each throat, and the effective gravitational coupling of the bag solution. This is the main technical result of the paper. To extract its physical significance we reexpress it in three different ways. In section 6 we comment on the relation to bimetric and multi-trace models, while section 7 contains some concluding remarks. Explicit expressions for the metric and dilaton of the 'bagpipes' solutions are collected in the appendix.

\section{Mass as holographic leakage}

We begin our discussion of massive AdS gravity from the dual CFT side. This sheds instructive light on the Higgsing mechanism and motivates the construction of the dual supergravity solutions. Recall that holographic duality associates to any $\mathrm{AdS}_{4}$ vacuum of string theory a three-dimensional conformal field theory $\left(\mathrm{CFT}_{3}\right)$. The $\mathrm{AdS}_{4}$ graviton is mapped to the energy-momentum tensor $T_{a b}$ of the $\mathrm{CFT}_{3}$, and the mass $\left(m_{\mathrm{g}}\right)$ of the former to the scaling dimension $\left(\Delta_{\mathrm{g}}\right)$ of the latter via [42]

$$
m_{\mathrm{g}}^{2} L_{4}^{2}=\Delta_{\mathrm{g}}\left(\Delta_{\mathrm{g}}-3\right)
$$

where $L_{4}$ is the $\mathrm{AdS}_{4}$ radius. ${ }^{3}$ The operator $T_{a b}$ and its tower of derivatives arrange themselves in a spin-2 highest-weight representation of the conformal algebra $\mathfrak{s o}(2,3)$. Usually the energy-momentum tensor is conserved, $\partial^{b} T_{a b}=0$, so this representation must be short since it has three null descendant states. A simple algebraic computation then shows that $T_{a b}$ must have canonical scaling dimension $\Delta_{\mathrm{g}}=3$, and the dual $\mathrm{AdS}_{4}$ graviton is hence massless.

To obtain a massive graviton we must therefore allow $3 d$ energy-momentum to 'leak out'. 4 There are two possibilities that are consistent with $\mathfrak{s o}(2,3)$ symmetry:

(i) Couple the original theory to another $3 d$ theory so that conformal symmetry is preserved. The coupling could be a double- trace deformation $[36,37]$, or it could be mediated by messenger degrees of freedom [1]. If it is weak the dual low-energy string theory is a bimetric theory, with one graviton massless and the other obtaining a small mass;

(ii) Consider the original theory as a defect or boundary of some higher-dimensional theory, in the simplest case a $\mathrm{CFT}_{4}$. The $3 d$ energy-momentum can now leak out in the extra dimension $\partial^{b} T_{a b}=\left.T_{a 4}\right|_{\text {defect }} \neq 0$. There is therefore now no shortening condition, and $T_{a b}$ acquires an anomalous dimension [47], $\epsilon=\Delta_{\mathrm{g}}-3>0$ (unitarity requires that it be non-negative).

\footnotetext{
${ }^{3} \mathrm{In}$ warped compactifications both $m_{\mathrm{g}}$ and $L_{4}$ vary in the transverse space, but their product is constant - see below.

${ }^{4}$ Leakage of $T_{a b}$ should not be confused with the transparent boundary conditions of [33, 34] on the gravity side.
} 
These two options are related - we will here focus on option (ii) which can be obtained as a limit of option (i). Since the graviton mass is proportional to the $3 d$ energy-momentum leakage, we want the latter to be weak. ${ }^{5}$ In principle this could be achieved by fine tuning a (nearly or exactly) marginal bulk-boundary coupling, but this is not the mechanism at work here. Weak leakage will be instead ensured by the scarcity of the bulk $\mathrm{CFT}_{4}$ degrees of freedom, as compared to those of the boundary $\mathrm{CFT}_{3}$. A consequence of this is that the Higgsing will not be a continuous process in these models, even though the graviton mass can be arbitrarily small.

Let us be now specific about the defect CFT. The natural candidate for the bulk $\mathrm{CFT}_{4}$ is $\mathcal{N}=4$ super Yang-Mills with gauge group $\mathrm{SU}(n)$ and coupling $g_{\mathrm{YM}}$. Its half -BPS superconformal boundaries and interfaces have been analyzed by Gaiotto and Witten [48]. Half-maximal supersymmetry guarantees the stability of the solutions, and gives extra technical control, but it is not otherwise essential. The graviton mass, in particular, is not a protected quantity as we will see in a minute. Weak leakage of $3 d$ energy-momentum could be achieved in the decoupling limit $g_{\mathrm{YM}} \rightarrow 0$, but this limit is singular. A better alternative is to insist that there are much fewer degrees of freedom in the bulk than on the boundary. We will indeed show in section 5 that the anomalous dimension of $T_{a b}$ scales like $\epsilon \sim n^{2} / \tilde{F}_{3}$, where $\tilde{F}_{3}$ is the free energy on the 3 -sphere which measures the boundary degrees of freedom [49].

\section{Recombination of representations}

Before moving to geometry, let us discuss the Higgsing from the point of view of representation theory. Let $[s]_{\Delta}$ denote a unitary highest-weight representation of $\mathfrak{s o}(2,3)$ with conformal primary of spin $s$ and scaling dimension $\Delta$. Massive gravitons belong to long representations of the algebra. The decomposition of a long spin- $s$ representation for $s \geq 1$ at the unitarity threshold reads [33, 34]

$$
[s]_{s+1+\epsilon} \stackrel{\epsilon \rightarrow 0}{\longrightarrow}[s]_{s+1} \oplus[s-1]_{s+2} .
$$

Thus the $\mathrm{AdS}_{4}$ graviton $(s=2)$ acquires a mass by eating a massive Goldstone vector. In the $10 d$ supergravity this vector must be that combination of off-diagonal components of the metric and tensor fields which is dual to the CFT operator $T_{a 4}$.

Since we will here deal with $\mathcal{N}=4$ backgrounds, supergravity fields and dual CFT operators fit in representations of the larger superconformal algebra osp $(4 \mid 4)$. These have been all classified under mild assumptions, see $[50,51]$ and references therein. In the notation of [51] (slightly retouched in [52]) the supersymmetric extension of the above $s=2$ decomposition reads

$$
L[0]_{1+\epsilon}^{(0 ; 0)} \stackrel{\epsilon \rightarrow 0}{\longrightarrow} A_{2}[0]_{1}^{(0 ; 0)} \oplus B_{1}[0]_{2}^{(1 ; 1)},
$$

where $L$ denotes a long representation, $A$ a short one at unitarity threshold, $B$ a short representation below threshold, and $[s]_{\Delta}^{\left(j ; j^{\prime}\right)}$ denotes a superconformal primary with spin

\footnotetext{
${ }^{5}$ In generic defect CFTs the defect-to-bulk leakage is very strong and $\Delta_{\mathrm{g}}-3 \sim O(1)$. In such cases there is no hope of any effective $4 d$ description on the gravity side.
} 
$s$, scaling dimension $\Delta$ and $\mathfrak{s o}(4)$ R-symmetry quantum numbers $\left(j ; j^{\prime}\right)$. The recombination (3.2) describes the Higgsing of the $\mathcal{N}=4$ graviton multiplet in $\mathrm{AdS}_{4}$. That this is at all possible is not automatic. For instance $\mathcal{N}=4$ supersymmetry forbids the Higgsing of ordinary gauge symmetries because conserved vector currents transform in absolutely protected representations of $\mathfrak{o s p}(4 \mid 4)[53,54]$.

The content of the conserved stress-tensor multiplet $A_{2}[0]_{1}^{(0 ; 0)}$, or equivalently the dual massless $\mathcal{N}=4$ supergraviton multiplet, is as follows:

$$
\begin{array}{cl}
\text { bosons : } & {[0]_{1}^{(0 ; 0)} \oplus[0]_{2}^{(0 ; 0)} \oplus[1]_{2}^{(1 ; 0) \oplus(0 ; 1)} \oplus[2]_{3}^{(0 ; 0)},} \\
\text { fermions : } & {[1 / 2]_{\frac{3}{2}}^{\left(\frac{1}{2} ; \frac{1}{2}\right)} \oplus[3 / 2]_{\frac{5}{2}}^{\left(\frac{1}{2} ; \frac{1}{2}\right)} .}
\end{array}
$$

In addition to the spin- 2 graviton, it includes four gravitini, six vectors, four spin- $1 / 2$ and two scalar fields, making a total of 16 bosonic and as many fermionic states. ${ }^{6}$ The Goldstone multiplet $B_{1}[0]_{2}^{(1 ; 1)}$ is much less familiar. Its content is as follows (see page 74 of $[51])$ :

$$
\begin{aligned}
& \text { bosons : } \quad[0]_{2}^{(1 ; 1)} \oplus[1]_{3}^{(1 ; 1) \oplus(1 ; 0) \oplus(0 ; 1)} \oplus[0]_{3}^{(2 ; 0) \oplus(0 ; 2) \oplus(1 ; 0) \oplus(0 ; 1) \oplus(1 ; 1) \oplus(0 ; 0)} \\
& \oplus[1]_{4}^{(0 ; 0) \oplus(1 ; 0) \oplus(0 ; 1)} \oplus[0]_{4}^{(1 ; 1) \oplus(0 ; 0)} \oplus[0]_{5}^{(0 ; 0)}, \\
& \text { fermions : } \quad[1 / 2]_{\frac{5}{2}}^{\left(\frac{3}{2} ; \frac{1}{2}\right) \oplus\left(\frac{1}{2} ; \frac{3}{2}\right) \oplus\left(\frac{1}{2} ; \frac{1}{2}\right)} \oplus[3 / 2]_{\frac{7}{2}}^{\left(\frac{1}{2} ; \frac{1}{2}\right)} \oplus[1 / 2]_{\frac{7}{2}}^{\left(\frac{3}{2} ; \frac{1}{2}\right) \oplus\left(\frac{1}{2} ; \frac{3}{2}\right) \oplus 2\left(\frac{1}{2} ; \frac{1}{2}\right)} \oplus[1 / 2]_{\frac{9}{2}}^{\left(\frac{1}{2} ; \frac{1}{2}\right)}
\end{aligned}
$$

Counting gives 112 physical bosonic fields and as many fermionic ones. The latter include four spin-3/2 fields which are not part of the spectrum of gauged $\mathcal{N}=4$ supergravity in four dimensions, as stressed in ref. [52]. The reader may be surprised that four extra spin- $3 / 2$ fields, rather than four spin- $1 / 2$ Goldstini, are here needed to make the gravitini massive. Note however that the unusual process at hand does not break the $\mathcal{N}=4$ supersymmetry, it instead makes the entire graviton supermultiplet massive. This is reminiscent of the familiar Higgsing of $\mathcal{N}=2$ vector multiplets, where the spin- $1 / 2$ gaugini obtain mass by combining with the spin- $1 / 2$ Higgsini.

\section{Scottish bagpipes}

We turn now to the gravity side of the Higgsing. The local form of all solutions of typeIIB supergravity with osp $(4 \mid 4)$ symmetry has been derived by D'Hoker et al. [24, 25] (see also $[55,56]$ for earlier work). Global solutions and the detailed holographic dictionary have been worked out in $[28,29,52]$. All solutions are warped products of $\mathrm{AdS}_{4}$ over a base manifold $\mathrm{M}_{6}$,

$$
d s_{10}^{2}=L_{4}^{2}(y) d s_{\mathrm{AdS}_{4}}^{2}+\sum_{i, j=1}^{6} g_{i j}(y) d y^{i} d y^{j},
$$

\footnotetext{
${ }^{6}$ The CFT operators include $T_{a b}$, six conserved R-symmetry currents and two scalar operators. Taking into account the conservation laws this gives also a total of $25-9=16$ independent operators.
} 


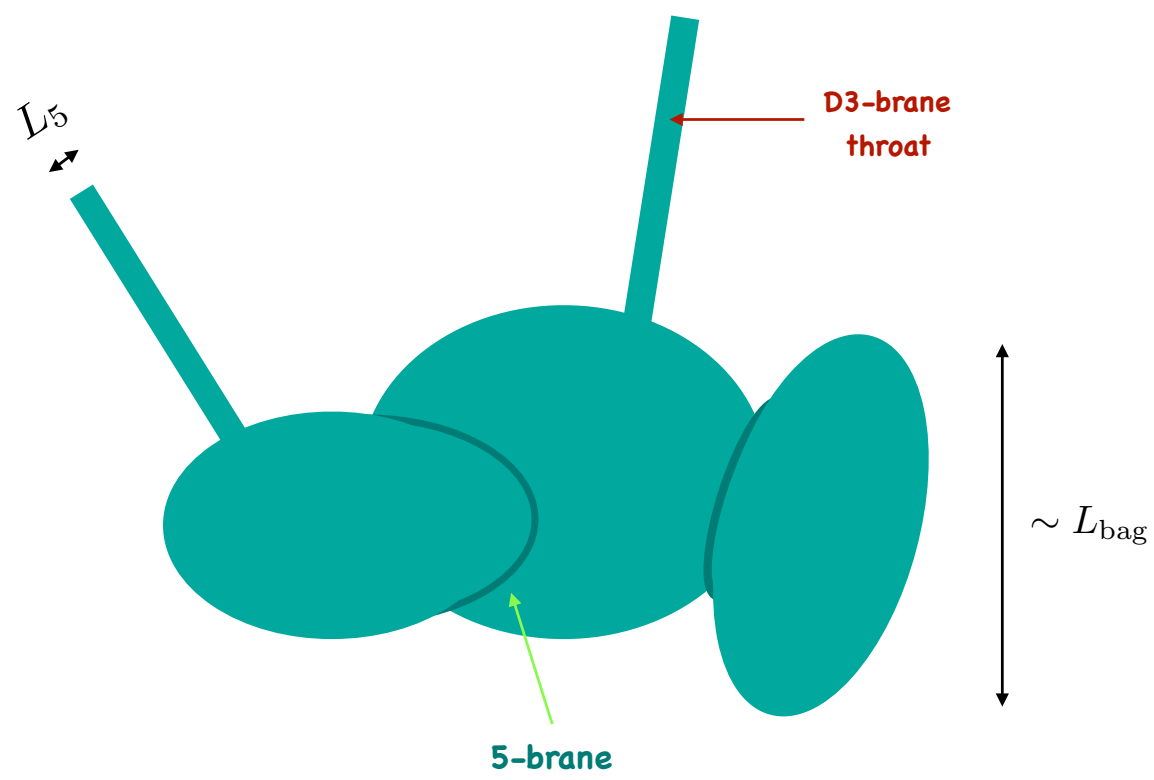

Figure 1. The 'bagpipes' manifold $\mathrm{M}_{6}$ consists of semiinfinite pipes with cross-sectional radius $L_{5}$, attached to a compact bag of typical size $\sim L_{\text {bag }} \gg L_{5}$. The dark curves on the bag depict 5-brane singularities. The $\mathrm{AdS}_{4}$ scale factor diverges at infinity in the pipes so that the full $10 d$ geometry asymptotes to $\left(\mathrm{AdS}_{5} / \mathbb{Z}_{2}\right) \times \mathrm{S}^{5}$.

where $d s_{\mathrm{AdS}_{4}}^{2}$ is the metric of the unit-radius Anti-de Sitter spacetime, $\left\{y^{i}\right\}$ are the coordinates of $\mathrm{M}_{6}$ with metric $g_{i j}$, and $L_{4}(y)$ is the local radius of the $\mathrm{AdS}_{4}$ fiber at a point $y$. The base manifold $\mathrm{M}_{6}$ is itself the warped product of two 2-spheres over a Riemann surface. The complete $\mathrm{AdS}_{4} \times \mathrm{S}^{2} \times \hat{\mathrm{S}}^{2}$ fiber realizes the $\mathfrak{s o}(2,3) \times \mathfrak{s o}(4) \subset \mathfrak{o s p}(4 \mid 4)$ bosonic symmetry of the backgrounds.

For the lightest $4 d$ graviton to acquire mass, $\mathrm{M}_{6}$ should not be a compact manifold. The manifolds that lead to a small graviton mass actually resemble six-dimensional Scottish bagpipes: they have one or more semi-infinite throats (the 'pipes') attached to a large central core (the 'bag') as illustrated in figure 1 . The full $10 d$ geometry of the pipes is $\mathrm{AdS}_{5} \times \mathrm{S}^{5}$, or its Janus generalization [25] in which the dilaton is also allowed to vary. A crucial technical remark $[27,28]$ is that for generic manifolds $\mathrm{M}_{6}$ (whose bags have both NS5-brane and D5-brane sources) the pipes can be shrank smoothly away ${ }^{7}$ leaving behind simple coordinate singularities. In this limit the bag reduces to a compact manifold $\overline{\mathrm{M}}_{6}$, and $\mathrm{AdS}_{4} \times_{w} \overline{\mathrm{M}}_{6}$ becomes a standard $\mathrm{AdS}_{4}$ vacuum with a massless $4 d$ graviton in its spectrum. Since we want the graviton to acquire a small mass, we will keep the throat radii finite but much smaller than the characteristic size of the bag.

The exact metric and dilaton backgrounds of the solutions are summarized in the appendix. $^{8}$ The bag depends on a set of integer D5-, NS5- and D3-brane charges which

\footnotetext{
${ }^{7}$ Strictly-speaking this is possible in the supergravity approximation. In string theory the throat radii are quantized.

${ }^{8}$ The supergravity solutions are actually singular at certain 2-cycles of $\mathrm{M}_{6}$ that are wrapped by D5- and NS5-branes. In their vicinity higher-order stringy and loop corrections cannot be neglected, but fortunately they do not affect the computation of the graviton mass at leading order in $L_{5} / L_{\mathrm{bag}}$.
} 
can be arranged in two Young tableaux [28]. Most of these will play however no role here. The only relevant bag parameters are (i) an overall measure of its size $\sim L_{\text {bag }}$ to be defined below, and (ii) the values of the dilaton at the entries of the throats. Note that the bag is a sort of 'composite Karch-Randall brane'. Tuning the available parameters to make it flat, as in ref. [22], makes it so thick that it ends up occupying (figuratively, not literally) most of space [26]. As stated above this is a facet of the scale non-separation problem of AdS vacua: $L_{4}$ is parametrically tied to the characteristic size of $\overline{\mathrm{M}}_{6}$.

The spectral problem for spin-2 excitations around any $\mathrm{AdS}_{4}$ supergravity solution was set up in ref. [26] (generalizing mildly [57]). Interestingly this problem only depends on the Einstein-frame metric, and not on the scalar and flux background fields. Mass eigenstates factorize as $\psi(y) \chi_{\mu \nu}$, where $\chi_{\mu \nu}$ is an eigenfunction of the wave operator in $\operatorname{AdS}_{4}$, i.e. $\mathcal{L}_{(2)}^{\mathrm{AdS}} \chi_{\mu \nu}=\lambda \chi_{\mu \nu}$ where $\mathcal{L}_{(2)}^{\mathrm{AdS}}$ is the (Lichnerowicz-Laplace) operator that acts on spin-2 (transverse-traceless) excitations, and the eigenvalue $\lambda$ is related to the mass via

$$
\lambda+2=m^{2}(y) L_{4}^{2}(y) .
$$

Note that both the mass and the $\mathrm{AdS}_{4}$ radius may vary as functions of the coordinates $y^{i}$, but their product is constant. It is this invariant squared mass that replaces the left-hand side of eq. (2.1) in warped (as opposed to direct-product) solutions.

The Kaluza-Klein mass spectrum is determined by the elliptic operator acting on the wavefunctions $\psi(y)$ on $\mathrm{M}_{6}[26]$

$$
\mathcal{M}^{2} \psi:=-\frac{L_{4}^{-2}}{\sqrt{g}} \partial_{i}\left(L_{4}^{4} \sqrt{g} g^{i j} \partial_{j} \psi\right)=(\lambda+2) \psi
$$

For direct-product solutions with constant $L_{4}, \mathcal{M}^{2}$ is simply the Laplace-Beltrami operator on $\mathrm{M}_{6}$. To define the spectral problem we need also to provide a norm. With canonically-normalized fields in ten dimensions ${ }^{9}$ the Kaluza-Klein reduction of the inner product reads [26]

$$
\left\langle\psi_{1} \mid \psi_{2}\right\rangle=\int_{\mathrm{M}_{6}} d^{6} y \sqrt{g} L_{4}^{2} \psi_{1}^{*} \psi_{2}
$$

The mass-squared operator (4.3) is thus hermitean and non-negative, as expected.

To summarize this section, we are interested in the smallest eigenvalue of the above mass operator, $\mathcal{M}^{2}$, for manifolds consisting of a large compact bag $\left(\overline{\mathrm{M}}_{6}\right)$ attached to one or more thin semi-infinite Janus throats. It actually turns out that the solutions studied here, whose CFT duals are $\mathcal{N}=4$ linear-quiver gauge theories, admit at most two semiinfinite throats. But more general backgrounds based on star-like quivers could have more throats. As will be clear, each throat makes a separate contribution to the squared mass of the graviton in the $L_{5} / L_{\text {bag }} \ll 1$ limit.

\footnotetext{
${ }^{9}$ In our convention $\psi$ has dimensions of the $10 d$ gravitational coupling $\kappa_{10} \sim[\text { mass }]^{4}$. An overall multiplicative constant in the norm is irrelevant as it drops out from the expression for the mass.
} 


\section{Mass from Janus throats}

The general spectral problem defined in (4.3) and (4.4) is a difficult one. But we are only interested in the smallest eigenvalue given by the equivalent minimization problem

$$
\lambda_{0}+2=\min _{\psi}\left[\int_{\mathrm{M}_{6}} d^{6} y \sqrt{g} L_{4}^{4}\left(g^{i j} \partial_{i} \psi^{\star} \partial_{j} \psi\right)\right] \quad \text { with } \int_{\mathrm{M}_{6}} d^{6} y \sqrt{g} L_{4}^{2}|\psi|^{2}=1 .
$$

Here $\lambda_{0}+2=\Delta_{\mathrm{g}}\left(\Delta_{\mathrm{g}}-3\right)$ is the lowest eigenvalue of $\mathcal{M}^{2}$, and the expression in square brackets is $\left\langle\psi\left|\mathcal{M}^{2}\right| \psi\right\rangle$ after an integration by parts. If $\mathrm{M}_{6}$ were replaced by the compact bag $\overline{\mathrm{M}}_{6}$ (obtained by truncating the pipes) the minimum would have been the constant wavefunction

$$
\psi_{0}(y)=\left(\int_{\overline{\mathrm{M}}_{6}} d^{6} y \sqrt{g} L_{4}^{2}\right)^{-1 / 2}:=\psi_{\mathrm{bag}}
$$

corresponding to a massless graviton. But the infinite pipes make the constant $\psi$ nonnormalizable. Indeed, $\sqrt{g} L_{4}^{2}$ reaches a minimum value $L_{5}^{8}$ inside the pipes, then blows up at infinity where the $10 d$ geometry asymptotes to that of (half) the boundary of $\mathrm{AdS}_{5}$. This is explained in the appendix and illustrated in figure 2. Normalizable wavefunctions should therefore go to zero inside the pipes. Furthermore, it is clear from eq. (5.1) that in order to minimize the mass $\psi$ should go to zero in the region of minimal $\sqrt{g} L_{4}^{4}$ where gradients have lower cost, as shown in figure 2 .

To make the argument precise, separate the manifold $\mathrm{M}_{6}$ in three parts: (I) the bag, (II) the infinite throats, and (III) the matching regions where throats are attached to the bag. Minimizing $\mathcal{M}^{2}$ in region (I) sets $\psi$ to a constant, so the bag does not contribute to the graviton mass. On the other hand, at leading order in $L_{5} / L_{\text {bag }}$ only the bag contributes to the norm of $\psi$. The reason is that $\sqrt{g} L_{4}^{2}$ decreases exponentially fast in the matching region, and $\psi$ vanishes exponentially fast in the throat as will be shown in a minute. This fixes the constant value $\psi_{0} \simeq \psi_{\text {bag }}$ in region (I).

The region of minimal $\sqrt{g} L_{4}^{4}$, on the other hand, where $\psi_{0}$ can vary with minimal mass cost, lies deep inside the throat regions. Only the throats will therefore contribute to the graviton mass at this leading order, an assumption whose validity will be again verified a posteriori.

In summary, the leading-order contribution to the norm comes from the bag, while the leading-order contribution to the mass comes from the bottom of the throat where $\sqrt{g} L_{4}^{4}$ is minimal. The matching region (III) contributes to neither and can be neglected. We may thus reformulate the problem as a variational problem in the Janus geometry:

$$
\lambda_{0}+2 \simeq \min _{\psi}\left[\int_{\text {throats }} d^{6} y \sqrt{g} L_{4}^{4} g^{i j} \partial_{i} \psi^{\star} \partial_{j} \psi\right] \text { with } \quad \psi \rightarrow \begin{cases}\psi_{\text {bag }} & \text { in matching region } \\ 0 & \text { at infinity }\end{cases}
$$

The only residual dependence on $\mathrm{M}_{6}$ (viz. on the composite NS5-D5-D3 brane) is via the boundary value $\psi_{\text {bag }}$ whose physical meaning will soon be made clear. 

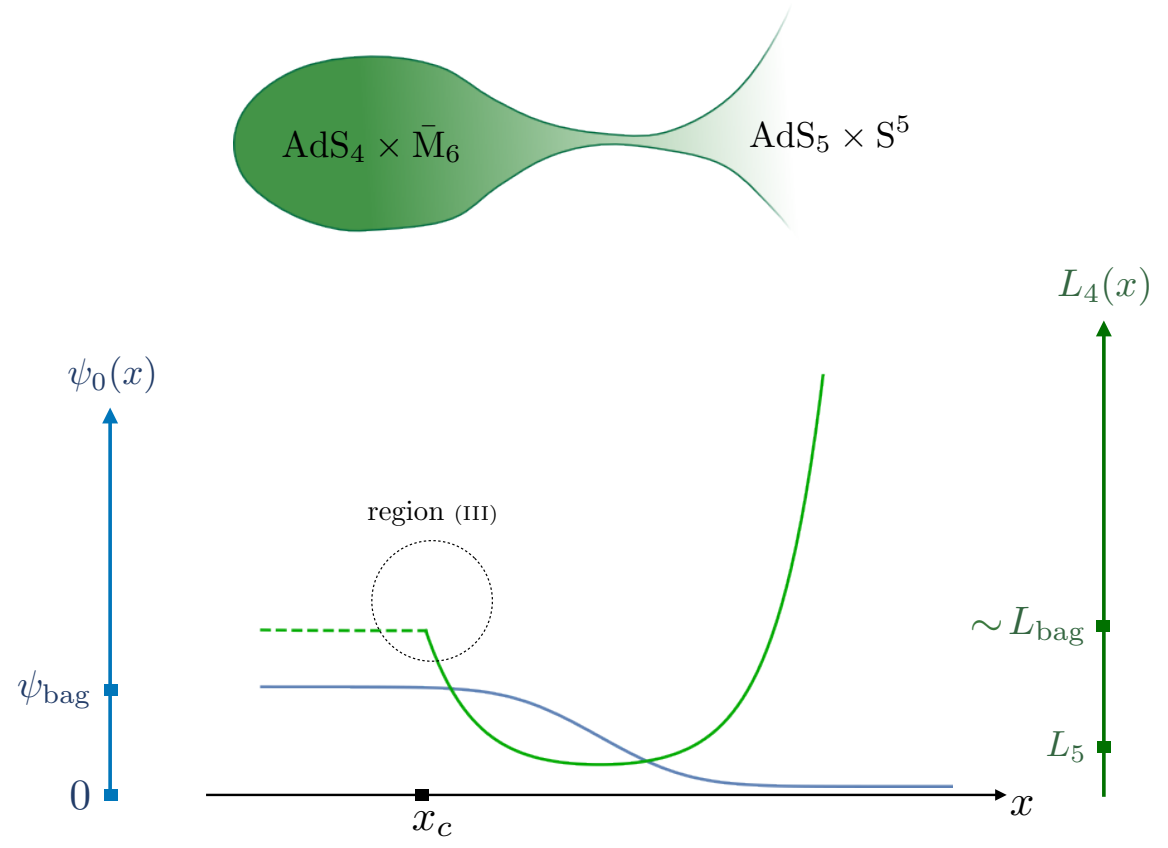

Figure 2. Schematic drawing of the $\mathrm{AdS}_{4}$ radius $L_{4}$ (green curve) and of the graviton wavefunction $\psi_{0}$ (blue curve) as functions of the coordinate $x$ of the Janus throat. The radius reaches a minimum value of $L_{5}$ inside the throat, and grows as $L_{5} \cosh x$ on either side where the geometry asymptotes to $\mathrm{AdS}_{5} / \mathbb{Z}_{2} \times \mathrm{S}^{5}$. The left half of the throat is cut-off by the 'bag' at a characteristic radius $\sim L_{\text {bag }} \gg$ $L_{5}$. The graviton wavefunction approaches a constant in this region, and vanishes exponentially at infinity. Since the matching region (III) contributes neither to the norm nor to the mass, it can be shrunk for our purposes to a point.

The $\mathcal{N}=4$ supersymmetric Janus solution [25] depends on two parameters, the radius $L_{5}$ and the dilaton variation $\delta \phi$. Like all other solutions in this class it has the fibered form

$$
d s_{10}^{2}=L_{4}^{2} d s_{\mathrm{AdS}_{4}}^{2}+d s_{\mathrm{M}_{6}}^{2} \quad \text { with } \quad d s_{\mathrm{M}_{6}}^{2}=f^{2} d s_{\mathrm{S}^{2}}^{2}+\hat{f}^{2} d s_{\hat{\mathrm{S}}^{2}}^{2}+4 \rho^{2} d z d \bar{z}
$$

The scale factors $L_{4}, f, \hat{f}, \rho$ depend on the complex coordinate $z$ that parametrizes the infinite strip. We write $z=x+i \tau$ with $\tau \in[0, \pi / 2]$. The metric factors and dilaton are given in the appendix. Here we only need the combination that enters in the square brackets in (5.3). Things simplify actually further because the spin-2 eigenfunctions in the Janus geometry factorize into spherical harmonics on the 2-spheres, and separate functions of $x$ and $\tau$, and the lightest mode is only function of $x$ [26]. Integrating over the 2-spheres and $\tau$ gives ${ }^{10}$

$$
\lambda_{0}+2=\min _{\psi}\left[\frac{\pi^{3}}{4} L_{5}^{8} \int_{x_{c}}^{\infty} d x \mathcal{G}(x)\left(\frac{d \psi}{d x}\right)^{2}\right] \quad \text { with } \quad \psi(x) \rightarrow \begin{cases}\psi_{\text {bag }} & \text { at } x=x_{c} \\ 0 & \text { at } x=\infty\end{cases}
$$

\footnotetext{
${ }^{10}$ When $\delta \phi=0$ the 2-spheres and the coordinate $\tau$ make up the 5 -sphere in $\operatorname{AdS}_{5} \times \mathrm{S}^{5}$, and $\mathcal{G}(x)=$ $4 \cosh ^{4} x$.
} 
where the function $\mathcal{G}(x)$, computed in the appendix, reads

$$
\mathcal{G}(x):=\left(\frac{\cosh 2 x+\cosh \delta \phi}{\cosh \delta \phi}\right)^{2} .
$$

We have cutoff the integral at some large negative value $x_{c}$, at the boundary of the matching region. The value of $x_{c}$ will drop out and could be replaced by $-\infty$, its only role is to remind us that $\psi$ would have been a non-normalizable mode in the complete Janus geometry.

The variational problem (5.5) can be easily solved,

$$
\frac{d}{d x}\left(\mathcal{G} \frac{d \psi_{0}}{d x}\right)=0 \Longrightarrow \psi_{0}(x)=c_{1}+c_{2} \int_{0}^{x} \frac{d x^{\prime}}{\mathcal{G}\left(x^{\prime}\right)}
$$

where $c_{1}, c_{2}$ are integration constants. We can perform the integral analytically with the result

$$
\begin{aligned}
I(x, a):= & \int_{0}^{x} \frac{a^{2} d x^{\prime}}{\left(\cosh 2 x^{\prime}+a\right)^{2}} \\
= & \frac{a^{3}}{2\left(a^{2}-1\right)^{3 / 2}} \log \left[\frac{\sqrt{a+1}+\sqrt{a-1} \tanh x}{\sqrt{a+1}-\sqrt{a-1} \tanh x}\right] \\
& -\frac{a^{2}}{\left(a^{2}-1\right)} \frac{\tanh x}{\left[(a+1)-(a-1) \tanh ^{2} x\right]} .
\end{aligned}
$$

We here set $\cosh \delta \phi=a$ and chose the lower integration limit so that $I$ is an odd function of $x$. Fixing $c_{1}, c_{2}$ so as to satisfy the boundary conditions (5.5) gives finally the graviton wavefunction in the throat

$$
\psi_{0}(x, a) \simeq \frac{1}{2} \psi_{\mathrm{bag}}\left[1-\frac{I(x, a)}{I(\infty, a)}\right] .
$$

Note that $I$ approaches its limiting values exponentially, so $\psi_{0}\left(x_{c}\right) \simeq \psi_{\text {bag }}$ up to exponentially small corrections. Furthermore at $x \rightarrow+\infty, \psi_{0}=O\left(e^{-2 x}\right)$ as required for the norm to be finite. The reader can now check that this contribution to the norm is parametrically smaller than that of the bag, and can be neglected as claimed earlier.

Plugging the above wavefunction in the expression (5.5) leads to the graviton mass. Note that $\psi_{0}$ obeys eq. (5.7), so the integrand is a total derivative and one finds

$$
\lambda_{0}+2=\frac{\pi^{3}}{4} L_{5}^{8} \int_{x_{c}}^{\infty} d x \mathcal{G}\left(\frac{d \psi_{0}}{d x}\right)^{2}=\frac{\pi^{3}}{4} L_{5}^{8}\left[\mathcal{G} \frac{d \psi_{0}}{d x} \psi_{0}\right]_{x_{c}}^{\infty} .
$$

Since $\mathcal{G}(x) d \psi_{0} / d x=-\psi_{\mathrm{bag}} / 2 I(\infty, a)$ and $\left[\psi_{0}\right]_{x_{c}}^{\infty}=-\psi_{\text {bag }}$, we finally get

$$
\lambda_{0}+2=\frac{\pi^{3}}{8} L_{5}^{8} \psi_{\text {bag }}^{2} / I(\infty, a)=\frac{3 \pi^{3}}{4} L_{5}^{8} \psi_{\text {bag }}^{2} J(a),
$$

where we have introduced the Janus correction factor $J(a)$,

$$
J(a)^{-1}:=6 I(\infty, a)=\frac{3 a^{3}}{\left(a^{2}-1\right)^{3 / 2}} \log \left[a+\sqrt{a^{2}-1}\right]-\frac{3 a^{2}}{\left(a^{2}-1\right)} .
$$




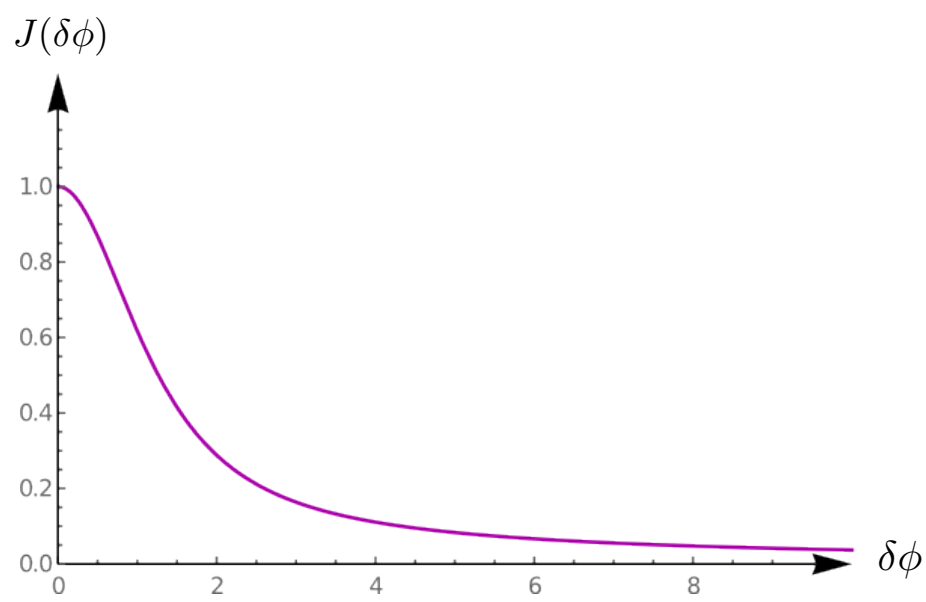

Figure 3. The Janus correction function, $J$, defined in equation (5.12).

As $a=\cosh \delta \phi$ ranges from 1 to $\infty, J(a)$ decreases monotonically from 1 to 0 , see figure 3 . The function is normalized so as to drop out for $\operatorname{AdS}_{5} \times S^{5}$ throats, while more generally it has the effect of reducing the graviton mass. This can be understood intuitively as follows: $\delta \phi$ is the difference between the value of the dilaton at the entry of the throat and its value at infinity. The former is fixed by the bag (see the appendix), while the latter is a free parameter that determines the coupling $g_{\mathrm{YM}}$ of the dual $4 d, \mathcal{N}=4$ super Yang-Mills theory.

Taking $g_{\mathrm{YM}}$ to zero (and hence $|\delta \phi| \rightarrow \infty$ ) decouples the bulk CFT, restores conservation of $T_{a b}$ and sends the graviton mass to zero. The same is true, by S-duality, if $g_{\mathrm{YM}}$ is taken to infinity - it is the bulk magnetic theory now that decouples. Both these limits are however singular. Not only does supergravity break eventually down, but also the spectrum in the Janus throat becomes quasi-continuous [26] invalidating any effective $4 d$ description.

Eqs. (5.11), (5.12) are the main technical result of this paper. To extract their physical meaning we will rewrite them in three different ways. We first express $\psi_{\text {bag }}$, defined in eq. (5.2), in terms of geometric data,

$$
\psi_{\mathrm{bag}}^{-2}=\int_{\overline{\mathrm{M}}_{6}} \sqrt{g} L_{4}^{2}=V_{6}\left\langle L_{4}^{2}\right\rangle_{\mathrm{bag}}
$$

where $V_{6}$ is the volume of the bag, and $\left\langle L_{4}^{2}\right\rangle_{\text {bag }}$ the average $\mathrm{AdS}_{4}$ squared radius (which is finite after truncating away the throats). The contribution (5.11) to the graviton mass thus reads ${ }^{11}$

$$
\text { geometric : } \quad m_{\mathrm{g}}^{2} L_{4}^{2}=\frac{3 \pi^{3} L_{5}^{8}}{4 V_{6}\left\langle L_{4}^{2}\right\rangle_{\mathrm{bag}}} \times J(\cosh \delta \phi)
$$

In the conventional $\mathrm{AdS}_{4} \times \bar{M}_{6}$ vacua the AdS radius is of the same order as the KaluzaKlein scale (this is again the scale non-separation problem), so $\left(V_{6}\right)^{1 / 3} \sim L_{\text {bag }}^{2} \sim\left\langle L_{4}^{2}\right\rangle_{\text {bag }}$.

\footnotetext{
${ }^{11}$ If there are more than one throats, one adds up their contributions on the right-hand-side.
} 
Thus $m_{\mathrm{g}}^{2}$ is suppressed by the eighth power of $L_{5} / L_{4}$, as compared to the second power which is the result in the thin-brane Karch-Randall model [58].

For a different rewriting, we use the relation between the compactification volume and the $4 d$ effective gravitational coupling, $\kappa_{10}^{2}=V_{6} \kappa_{4}^{2}$ where $\kappa_{10}$ is the coupling in ten dimensions. Using also the relations $2 \kappa_{10}^{2}=(2 \pi)^{7} \alpha^{\prime}{ }^{4} \lambda_{s}^{2}$ and $L_{5}^{4}=4 \pi \alpha^{\prime 2} \lambda_{s} n$, where $\alpha^{\prime}$ is the Regge slope, $\lambda_{s}$ the string coupling constant (which can be absorbed in the definition of the Einstein-frame metric) and $n$ the quantized D3-brane charge of the throat [59] we get

$$
\text { gravitational : } \quad m_{\mathrm{g}}^{2} L_{4}^{2}=\frac{3 n^{2}}{16 \pi^{2}} \frac{\kappa_{4}^{2}}{\left\langle L_{4}^{2}\right\rangle_{\mathrm{bag}}} \times J(\cosh \delta \phi) .
$$

This rewriting in terms of four-dimensional parameters brings forth two important points. First, the graviton mass is quantized so the Higgsing cannot be continuous [1]. To trust the supergravity approximation we need $n \gg 1$. Of course the mass does vanish with the continuous parameters $\kappa_{4}$ and $(\cosh \delta \phi)^{-1}$, but these limits as noted above are singular.

The second remark is that (5.15) has the same parametric form as the result found in refs. [33-35]. In these references the mass is a quantum effect arising from integrating out matter fields, while in our case it arose from a standard small-fluctuation analysis around a classical supergravity solution. We will comment on this again in the next section.

One final rewriting sheds further light on the dual CFT side. We argued earlier that what ensures a weak $3 d$ energy-momentum leakage is the scarcity of the bulk- $\mathrm{CFT}_{4}$ degrees freedom as compared to those on the $3 d$ defect. A measure of CFT degrees of freedom for even dimensions is the familiar Weyl-anomaly coefficient $a$, which for $\mathcal{N}=4$ super Yang Mills with gauge group $\mathrm{U}(n)$ is equal to $n^{2}$ [in the normalization in which the contribution of a scalar to $a$ is $1 / 90$ ]. The analog of $a$ in three dimensions is (minus) the free energy on $\mathrm{S}^{3}$, which is related to the vacuum entanglement entropy across a spatial circle [60,61]-[65]. These measures have been unified by Giombi and Klebanov in a generalized free energy [49] defined (via dimensional regularization) for arbitrary $d, \tilde{F}_{d}=\sin (\pi d / 2) \log Z\left(\mathrm{~S}^{d}\right)$. For even dimensions $\tilde{F}_{d}=(-)^{d / 2} a \pi / 2$, while in three dimensions a holographic calculation gives $\tilde{F}_{3}=4 \pi^{2}\left\langle L_{4}^{2}\right\rangle / \kappa_{4}^{2}$ (see for example section 4.2 of [66]). ${ }^{12}$ Combining everything we find

$$
\text { defect } \underline{\mathrm{CFT}}: \quad 3\left(\Delta_{\mathrm{g}}-3\right) \simeq m_{\mathrm{g}}^{2} L_{4}^{2}=\frac{6 \pi^{3} \tilde{F}_{4}}{\widetilde{F}_{3}} \times J(\cosh \delta \phi)
$$

where $\Delta_{\mathrm{g}}$ is the anomalous dimension of the almost conserved $3 d$ energy-momentum tensor. This way of expressing the result makes it clear that the expansion parameter is the ratio of generalized free energies between the bulk and the defect CFT.

\section{$6 \quad$ Bimetric and double-trace models}

In ref. [1] we analyzed solutions with a highly-curved $\mathrm{AdS}_{5} \times \mathrm{S}^{5}$ throat ${ }^{13}$ capped-off at both of its ends by bags of much larger size. The low-energy theory is in this case a two-graviton

\footnotetext{
${ }^{12}$ The CFT calculation has been performed using localization in refs. [67, 68].

${ }^{13}$ The restriction to $\mathrm{AdS}_{5} \times \mathrm{S}^{5}$ throats is valid for identical bags, or more generally for bags with the same value of the dilaton at the two throat entries. This was implicitly assumed in ref. [1].
} 
theory. This is a concrete realization of the idea of 'Weakly Coupled Worlds' [69] in which two or more Universes endowed with separate metrics are coupled through the mixing of their gravitational fields. It is well known that massive gravity can be obtained from bigravity in a decoupling limit, and this is also true for our solutions. Before exhibiting this decoupling limit, we will first generalize the analysis of [1] from $\mathrm{AdS}_{5} \times \mathrm{S}^{5}$ to Janus throats.

The manifold $\mathrm{M}_{6}$ now consists of a Janus throat capped-off on both sides by two bags, $\overline{\mathrm{M}}_{6}$ and $\overline{\mathrm{M}}_{6}{ }^{\prime}$. For economy of notation we introduce the parameters

$$
v:=\int_{\overline{\mathrm{M}}_{6}} \sqrt{g} L_{4}^{2} \quad \text { and } \quad v^{\prime}:=\int_{\overline{\mathrm{M}}_{6}^{\prime}} \sqrt{g} L_{4}^{2} .
$$

Note that $v$ is just a short-hand for the parameter $V_{6}\left\langle L_{4}^{2}\right\rangle_{\text {bag }}=\psi_{\text {bag }}^{-1 / 2}$ of the previous section. Using the inner product $\left\langle\psi_{1} \mid \psi_{2}\right\rangle=\int_{\mathrm{M}_{6}} \sqrt{g} L_{4}^{2} \psi_{1}^{*} \psi_{2}$ one finds easily two orthogonal, lowlying spin-2 states. A massless state with constant wavefunction throughout $\mathrm{M}_{6}$ (which is normalizable because $\mathrm{M}_{6}$ is now compact), and a massive state whose wavefunction is approximately constant in the bags,

$$
\psi_{0}(x) \simeq\left(v+v^{\prime}\right)^{-1 / 2} \times\left\{\begin{array}{cl}
\sqrt{v^{\prime} / v} & \text { in } \overline{\mathrm{M}}_{6}, \\
-\sqrt{v / v^{\prime}} & \text { in } \overline{\mathrm{M}}_{6}^{\prime} .
\end{array}\right.
$$

Since the throat makes a subleading contribution to the inner product, the above wavefunction is clearly orthogonal to the constant one, i.e. to the wavefunction of the massless graviton. This second mode is necessarily massive because $\psi_{0}$ is forced to vary inside the Janus throat in order to extrapolate between the above values at the exits.

One can now repeat almost verbatim the calculation of the previous section. The wavefunction in the Janus throat with the new boundary conditions reads

$$
\psi_{0} \simeq \frac{1}{2 \sqrt{v v^{\prime}\left(v-v^{\prime}\right)}}\left[\left(v^{\prime}-v\right)-\left(v^{\prime}+v\right) \frac{I(x, a)}{I(\infty, a)}\right]
$$

where $I(x, a)$ has been defined in eq. (5.8). Inserting the above wavefunction in (5.10), and reexpressing $v$ and $v^{\prime}$ in terms of radii and effective couplings gives

$$
m_{\mathrm{g}}^{2} L_{4}^{2}=\frac{3 n^{2}}{16 \pi^{2}}\left[\frac{\kappa_{4}^{2}}{\left\langle L_{4}^{2}\right\rangle_{\mathrm{bag}}}+\frac{\kappa_{4}^{2 \prime}}{\left\langle L_{4}^{2}\right\rangle_{\mathrm{bag}^{\prime}}}\right] \times J(\cosh \delta \phi) .
$$

This agrees with the result derived in [1] for pure $\operatorname{AdS}_{5} \times S^{5}$ throats for which $J=1 .{ }^{14}$ It also reduces to our formula of the previous section in the decoupling limit $v^{\prime} \rightarrow \infty$, i.e. $\kappa_{4}^{\prime} \rightarrow 0$ or equivalently $\left\langle L_{4}^{2}\right\rangle_{\mathrm{bag}^{\prime}} \rightarrow \infty$. In this limit the massless graviton has vanishing wavefunction and decouples, whereas $\psi_{0}$ is concentrated entirely in the (unprimed) bag $\overline{\mathrm{M}}_{6}$ and in the throat.

From the perspective of the dual field theory, these bigravity solutions are not $4 d$ defect CFTs, but rather $3 d$ CFTs of a special kind. They are superconformal gauge theories

\footnotetext{
${ }^{14}$ In this reference we worked in units of $L_{4}$, and absorbed $\left\langle L_{4}^{2}\right\rangle$ in the definition of $\kappa_{4}^{2}$. We thank Thibault Damour for insisting that we reestablish the units.
} 
based on linear quivers with a low-rank 'weak' node [1]. Removing this node breaks the quiver into two disjoint quivers. One could in principle integrate out the scarce messenger fields, thereby generating multitrace couplings between disjoint theories in the spirit of $[36,37]$. In contrast with these references, the couplings are however non-local (they are generated by massless messengers) and exactly scale invariant (the $\mathrm{AdS}_{4}$ symmetry is manifest). Conversely, integrating back in the messenger fields restores the interpretation of the multitrace couplings in terms of a classical supergravity background, and resolves the conflicts with string-theory locality discussed in refs. [39, 40].

Similar comments apply to the relation of our models with the transparent boundary conditions of [33-35]. These could conceivably mimic the effects of the semi-infinite throats, but they are obscuring the issues of locality and scale invariance. It is nevertheless interesting that they lead to the same parametric dependence of $m_{\mathrm{g}}$ on the effective gravitational coupling $\kappa_{4}$.

\section{Concluding remarks}

As these top-down embeddings demonstrate, massive $\mathrm{AdS}_{4}$ gravity is part of the stringtheory landscape. Since string theory is believed to be a consistent theory, we expect the effective $4 d$ low-energy gravity to be free of any pathologies. We have explained why the effective theory must break down at the AdS radius $L_{4}$, which is comparable to the KaluzaKlein scale. This is related to the scale non-separation problem and could be a generic feature of all such embeddings. Still there is a range of energies, $m_{\mathrm{g}}<E<L_{4}^{-1}$, in which one could try to compare with the effective actions of refs. $[7,8,10,11]$, and to explore in particular the possible onset of strong coupling.

In general, the low-energy theory contains massless gauge fields and a plethora of scalar moduli, and is hence far from minimal [52]. Nevertheless, the main actor for our purposes here is the Goldstone multiplet $B_{1}[0]_{2}^{(1 ; 1)}$ which furnishes the missing degrees of freedom of the massive $\mathcal{N}=4$ supergraviton. This multiplet includes four $4 \mathrm{~d}$ spin- $3 / 2$ excitations of the 10d gravitini, which acquire Kaluza-Klein mass via the compactification on the bag $\bar{M}_{6}$. While computing the interactions of these Goldstone modes directly from ten dimensions is in principle doable, in practice this is a formidable task.

One possible strategy could be to exploit the remarkable fact that the massive $\mathcal{N}=$ 4 supergraviton of eq. (3.2) has the same spin-3/2 degrees of freedom as the massless $\mathcal{N}=8$ supergraviton. This suggests that the above Higgsing process might admit a fourdimensional description as an $\mathcal{N}=4$ deformation of the maximal $\mathcal{N}=8$ supergravity of Cremmer, Julia and Scherk. ${ }^{15}$ Note that since $B_{1}[0]_{2}^{(1 ; 1)}$ contains extra gravitini, it cannot be part of the spectrum of any other, $\mathcal{N}<8$, two-derivative supergravity in four dimensions. Interestingly, extra degrees of freedom could be also obtained by adding higher-derivative terms, as was done recently in [79]. The authors of this reference analyzed the spectrum

\footnotetext{
${ }^{15}$ This possibility arose in a discussion with Pierre Fayet. Since parts of the Goldstone multiplet have masses of the order of the cutoff scale $L_{4}^{-1}$, this cannot be strictly-speaking a low-energy effective action, but at best a minimal completion thereoff that preserves $\mathcal{N}=4$ supersymmetry.
} 
around Minkowski spacetime and found it to contain ghosts. It might be interesting to repeat their analysis around Anti-de Sitter and see if there is any contact with our results.

Massive Poincaré gravity is harder to embedd in string theory and could possibly lie in swampland. One way to see the difficulty is as follows: a key feature of the Karch-Randall model is the existence of a local minimum of the AdS scale factor $L_{4}$. The existence of a minimum seems however to be in tension with the holographic $c$-theorem when the $\mathrm{AdS}_{4}$ fiber is replaced by Minkowski [22, 70, 71]. It is an open question whether this obstruction can be somehow relaxed.

In a different direction one can look for massive-gravity and bimetric models in other dimensions and/or with different amounts of supersymmetry. Many exact $\operatorname{AdS}_{D}$ solutions with $D>4$ and half-maximal supersymmetry are known by now, for instance [72] for $\mathrm{AdS}_{7},[73]-[76]$ for $\mathrm{AdS}_{6}$, and [77] for $\mathrm{AdS}_{5}$ (for a review and more references see also [78]). Some cases can be a priori excluded. A prime example is $6 d \mathcal{N}=1$, where the stress tensor belongs to a protected $B$-series multiplet [51] and cannot acquire an anomalous dimension. Thus massive $\mathrm{AdS}_{7}$ supergravity is a priori excluded. ${ }^{16}$ The stress tensor multiplet is also absolutely protected for $\mathcal{N}=1$ in $5 d$, and for more-than-half-maximal supersymmetry $(\mathcal{N}>2$ in $4 d$ and $\mathcal{N}>4$ in $3 d)$. This is consistent with the fact that there exist no candidate defect CFTs with so many unbroken supersymmetries. ${ }^{17}$ A situation with no protection is $\mathcal{N}=2 \mathrm{AdS}_{5}$. It would be interesting to search for embeddings of massive AdS supergravity or bigravity in this case. It would be even more interesting to search for non-supersymmetric embeddings that allow a Kaluza-Klein cutoff $\gg L_{4}$ along the lines of [30].

\section{Acknowledgments}

We have benefited from discussions or email exchanges with Laura Bernard, Ali Chamseddine, Cedric Deffayet, Eric D'Hoker, Gregory Gabadadze, Dieter Lüst, Slava Mukhanov, Alessandro Tomassielo and Christoph Uhlemann. We thank particularly Pierre Fayet for a discussion of supersymmetric Higgsing. C.B. aknowledges the hospitality of the Mani L. Bhaumik Institute of UCLA during the last stage of this work.

\section{A The type IIB solutions}

Here we collect some formulae on the exact type-IIB supergravity solutions that we use. For more extensive descriptions of this class of solutions see refs. [24, 25] and refs. [28, 29].

All solutions of type-IIB supergravity preserving $\mathcal{N}=4 \mathrm{AdS}_{4}$ supersymmetries have the form of a fibration over a Riemann-surface $\Sigma$,

$$
d s_{10}^{2}=L_{4}^{2} d s_{\mathrm{AdS}_{4}}^{2}+f^{2} d s_{\mathrm{S}^{2}}^{2}+\hat{f}^{2} d s_{\hat{\mathrm{S}}^{2}}^{2}+4 \rho^{2} d z d \bar{z}
$$

\footnotetext{
${ }^{16}$ The solutions of [72] resemble those studied here - they are dual to gauge theories based on linear quivers. Anomaly cancellation forces however these latter to be balanced quivers. This implies that the gauge-group rank is a concave function along the quiver, which forbids the low-rank 'bridge' nodes of [1].

${ }^{17}$ The relation of anomalous dimensions with charge leakage also implies that whenever the stress-tensor multiplet is protected, so are all conserved-current multiplets. Indeed in a local QFT no charge can leak out without carrying also some energy. Inspection of all superconformal representations in [51] confirms the validity of this assertion.
} 
where the scale factors of the three (pseudo)spheres depend only on the coordinate $z=$ $x+i \tau$ of the Riemann surface. For us this latter is the infinite strip, $0 \leq \tau \leq \pi / 2$. Solutions based on the annulus do not allow the attachment of semi-infinite Janus throats, and there are no known solutions based on higher-genus Riemann surfaces. The six-dimensional manifold $\mathrm{S}^{2} \times \hat{\mathrm{S}}^{2} \times \Sigma$ is called $\mathrm{M}_{6}$ in the main text (or $\overline{\mathrm{M}}_{6}$ when it is compact).

The expressions for the metric factors and the dilaton, $\phi$, depend on a pair of harmonic functions

$$
L_{4}^{8}=16 \frac{\mathcal{U} \hat{\mathcal{U}}}{W^{2}}, \quad f^{8}=16 h^{8} \frac{\hat{\mathcal{U}} W^{2}}{\mathcal{U}^{3}}, \quad \hat{f}^{8}=16 \hat{h}^{8} \frac{\mathcal{U} W^{2}}{\hat{\mathcal{U}}^{3}}, \quad \rho^{8}=\frac{\mathcal{U} \hat{\mathcal{U}} W^{2}}{h^{4} \hat{h}^{4}}, \quad e^{4 \phi}=\frac{\hat{\mathcal{U}}}{\mathcal{U}},
$$

where $h$ and $\hat{h}$ are the two harmonic functions, from which one computes ${ }^{18}$

$$
\mathcal{U}=2 h \hat{h}\left|\partial_{z} h\right|^{2}-h^{2} W, \quad \hat{\mathcal{U}}=2 h \hat{h}\left|\partial_{z} \hat{h}\right|^{2}-\hat{h}^{2} W, \quad \text { and } \quad W=\partial_{z} \partial_{\bar{z}}(h \hat{h}) .
$$

The solutions also have 5-form and 3-form fluxes whose explicit form we won't need.

After imposing regularity conditions, the most general solutions with the strip as base manifold are given by the following choice for the harmonic functions:

$$
\begin{aligned}
& h=-i \alpha \sinh (z-\beta)-\sum_{a=1}^{N} \gamma_{a} \log \tanh \left(\frac{i \pi}{4}-\frac{z}{2}+\frac{\delta_{a}}{2}\right)+\text { c.c. }, \\
& \hat{h}=\hat{\alpha} \cosh (z-\hat{\beta})-\sum_{b=1}^{\hat{N}} \hat{\gamma}_{b} \log \tanh \left(\frac{z}{2}-\frac{\hat{\delta}_{b}}{2}\right)+\text { c.c. }
\end{aligned}
$$

All parameters in these expressions are real. Furthermore the parameters $\left\{\alpha, \gamma_{a}\right\}$ and $\left\{\hat{\alpha}, \hat{\gamma}_{b}\right\}$ must all have the same sign which can be chosen positive - the harmonic functions are then positive everywhere inside the strip. The logarithmic singularities on the upper (lower) boundary of the strip correspond to D5-brane (NS5-brane) sources wrapping 2sphere cycles. The regions $z \rightarrow \pm \infty$, on the other hand, correspond to semi-infinite D3brane throats with the geometry of the supersymmetric Janus solution.

The pure Janus solution is found by setting $\gamma_{a}=\hat{\gamma}_{b}=0$. Its radius is $L_{5}=$ $2(\alpha \hat{\alpha} \cosh \delta \phi)^{1 / 4}$, where $\delta \phi=\beta-\hat{\beta}$ is the change of the dilaton as $x$ goes from $-\infty$ to $\infty$. By shifting the origin of the $x$ axis one may choose $\beta=-\hat{\beta}=\delta \phi / 2$. The combination of scale factors that enters in the expression (5.3) for the squared mass is

$$
\sqrt{g} L_{4}^{4} \rho^{-2}=L_{4}^{4} f^{2} \hat{f}^{2}=16 h^{2} \hat{h}^{2} .
$$

Inserting the Janus harmonic functions gives $16 h^{2} \hat{h}^{2}=\left(L_{5}^{8} / 16\right) \sin ^{2}(2 \tau) \mathcal{G}(x)$ where the function $\mathcal{G}$ is the one defined in eq. (5.6). Using finally the $\tau$-independence of $\psi_{0}$ [26], and integrating over the two 2-spheres and over $\tau$, leads to the expression (5.5) for the mass.

A typical 'scottish bagpipes' manifolds $\mathrm{M}_{6}$ has $\alpha, \hat{\alpha} \ll \gamma_{a}, \hat{\gamma}_{b}$ and all other parameters $\sim O(1)$. The bag $\overline{\mathrm{M}}_{6}$ is obtained in the limit $\alpha, \hat{\alpha}=0$ which truncates away the asymptotic

\footnotetext{
${ }^{18}$ In the previous literature $h, \hat{h}$ are denoted $h_{1}, h_{2}$ (and similarly for the associated functions $\mathcal{U}, \hat{\mathcal{U}}$, the 2-spheres $\mathrm{S}^{2}, \hat{\mathrm{S}}^{2}$, and the parameters $\alpha, \hat{\alpha}$ and $\beta, \hat{\beta}$ ). We think that the benefit of making S-duality (or mirror symmetry) manifest outweighs the risk of confusion from this change of notation .
} 
$\mathrm{AdS}_{5} / \mathbb{Z}_{2} \times \mathrm{S}^{5}$ regions of the Janus throats. It can be checked that $x= \pm \infty$ become simple coordinate singularities in this limit, and that $\overline{\mathrm{M}}_{6}$ is compact [28]. The product $\alpha \hat{\alpha}$ that controls the graviton Higgsing is a continuous parameter in supergravity, but it is quantized in string theory where it is related to the D3-brane charges of the throats (see below).

The detailed shape of the bag depends on the parameters $\left\{\gamma_{a}, \hat{\gamma}_{b}, \delta_{a}, \hat{\delta}_{b}\right\}$ which are in one-to-one corerspondence with the NS5-, D5- and D3-brane charges [28] of the 'fat KarchRandall brane'. In general the bag has many different length scales, but a typical size is $L_{\text {bag }} \sim\left(\sum \gamma_{a} \sum \hat{\gamma}_{b}\right)^{1 / 4}$ where the sum runs over 5-brane singularities in some $\delta z \sim O(1)$ region of the strip. In order to stabilize the bag one needs both NS5-brane and D5-brane charges, or else the dilaton runs away and the solution is singular.

Most of the details of the bag play no role in the calculation of the graviton mass at leading order. The only relevant parameters are $\psi_{\text {bag }}$ defined in eq. (5.2), and the four combinations

$$
\gamma_{ \pm}:=\sum_{a=1}^{N} \gamma_{a} e^{ \pm \delta_{a}} \quad \text { and } \quad \hat{\gamma}_{ \pm}:=\sum_{b=1}^{\hat{N}} \hat{\gamma}_{b} e^{ \pm \hat{\delta}_{b}}
$$

To see why let us divide the strip in several regions. For $x \sim O(1)$ we may neglect $\alpha, \hat{\alpha}$ altogether. This is the bag region (I) of section 5. For $x$ sufficiently large, on the other hand, we can expand the log tanh functions, and approximate $h$ and $\hat{h}$ as follows

$$
2 h \simeq-i\left(\alpha e^{z-\beta}-4 \gamma_{+} e^{-z}\right)+\text { c.c. }, \quad 2 \hat{h} \simeq\left(\hat{\alpha} e^{z-\hat{\beta}}+4 \hat{\gamma}_{+} e^{-z}\right)+\text { c.c. } .
$$

This is the throat region (II) of section 5, where the background is the supersymmetric Janus solution. Expressing (A.7) in terms of hyperbolic sine and cosine leads to the Janusparameter identification

$$
L_{5}^{(+)}=2 \sqrt{2}\left[\alpha \hat{\alpha} \gamma_{+} \hat{\gamma}_{+} e^{-(\beta+\hat{\beta})} \cosh ^{2} \delta \phi^{(+)}\right]^{1 / 8} \quad \text { and } \quad e^{2 \delta \phi^{(+)}}=\frac{\hat{\alpha} \gamma_{+}}{\alpha \hat{\gamma}_{+}} e^{\beta-\hat{\beta}} .
$$

Similar formulae hold for the parameters $L_{5}^{(-)}, \delta \phi^{(-)}$of the other throat, in the region $x \rightarrow-\infty$. The only thing to retain from this discussion is that for a given bag (i.e. given $\left.\gamma_{ \pm}, \hat{\gamma}_{ \pm}\right)$we have enough free parameters to choose the radii and dilaton jumps of the two asymptotic Janus throats at will.

Open Access. This article is distributed under the terms of the Creative Commons Attribution License (CC-BY 4.0), which permits any use, distribution and reproduction in any medium, provided the original author(s) and source are credited.

\section{References}

[1] C. Bachas and I. Lavdas, Quantum gates to other universes, Fortsch. Phys. 66 (2018) 1700096 [arXiv: 1711.11372] [INSPIRE].

[2] M. Fierz and W. Pauli, On relativistic wave equations for particles of arbitrary spin in an electromagnetic field, Proc. Roy. Soc. Lond. A 173 (1939) 211 [inSPIRE]. 
[3] K. Hinterbichler, Theoretical aspects of massive gravity, Rev. Mod. Phys. 84 (2012) 671 [arXiv:1105.3735] [INSPIRE].

[4] C. de Rham, Massive gravity, Living Rev. Rel. 17 (2014) 7 [arXiv:1401.4173] [INSPIRE].

[5] A. Schmidt-May and M. von Strauss, Recent developments in bimetric theory, J. Phys. A 49 (2016) 183001 [arXiv:1512.00021] [INSPIRE].

[6] D.G. Boulware and S. Deser, Can gravitation have a finite range?, Phys. Rev. D 6 (1972) 3368 [INSPIRE].

[7] C. de Rham and G. Gabadadze, Generalization of the Fierz-Pauli action, Phys. Rev. D 82 (2010) 044020 [arXiv: 1007.0443] [INSPIRE].

[8] C. de Rham, G. Gabadadze and A.J. Tolley, Resummation of massive gravity, Phys. Rev. Lett. 106 (2011) 231101 [arXiv: 1011.1232] [INSPIRE].

[9] S.F. Hassan and R.A. Rosen, Resolving the ghost problem in non-linear massive gravity, Phys. Rev. Lett. 108 (2012) 041101 [arXiv:1106.3344] [INSPIRE].

[10] A.H. Chamseddine and V. Mukhanov, Ghost free mimetic massive gravity, JHEP 06 (2018) 060 [arXiv: 1805. 06283] [INSPIRE].

[11] A.H. Chamseddine and V. Mukhanov, Mimetic massive gravity: beyond linear approximation, JHEP 06 (2018) 062 [arXiv: 1805.06598] [INSPIRE].

[12] N. Arkani-Hamed, H. Georgi and M.D. Schwartz, Effective field theory for massive gravitons and gravity in theory space, Annals Phys. 305 (2003) 96 [hep-th/0210184] [INSPIRE].

[13] A.H. Chamseddine and V. Mukhanov, Hidden ghost in massive gravity, JHEP 03 (2013) 092 [arXiv: 1302.4367] [INSPIRE].

[14] S. Deser, K. Izumi, Y.C. Ong and A. Waldron, Superluminal propagation and acausality of nonlinear massive gravity, arXiv:1312.1115 [INSPIRE].

[15] S. Deser, K. Izumi, Y.C. Ong and A. Waldron, Problems of massive gravities, Mod. Phys. Lett. A 30 (2015) 1540006 [arXiv:1410.2289] [INSPIRE].

[16] S.F. Hassan and M. Kocic, On the local structure of spacetime in ghost-free bimetric theory and massive gravity, JHEP 05 (2018) 099 [arXiv: 1706.07806] [INSPIRE].

[17] B. Bellazzini, F. Riva, J. Serra and F. Sgarlata, Beyond positivity bounds and the fate of massive gravity, Phys. Rev. Lett. 120 (2018) 161101 [arXiv:1710.02539] [INSPIRE].

[18] C. de Rham, S. Melville, A.J. Tolley and S.-Y. Zhou, Positivity bounds for massive spin-1 and spin-2 fields, arXiv:1804.10624 [INSPIRE].

[19] I.I. Kogan, S. Mouslopoulos and A. Papazoglou, The $m \rightarrow 0$ limit for massive graviton in $d S_{4}$ and $A d S_{4}$ : how to circumvent the van Dam-Veltman-Zakharov discontinuity, Phys. Lett. B 503 (2001) 173 [hep-th/0011138] [INSPIRE].

[20] M. Porrati, No van Dam-Veltman-Zakharov discontinuity in AdS space, Phys. Lett. B 498 (2001) 92 [hep-th/0011152] [INSPIRE].

[21] A.I. Vainshtein, To the problem of nonvanishing gravitation mass, Phys. Lett. B 39 (1972) 393.

[22] A. Karch and L. Randall, Locally localized gravity, JHEP 05 (2001) 008 [hep-th/0011156] [INSPIRE]. 
[23] A. Karch and L. Randall, Localized gravity in string theory, Phys. Rev. Lett. 87 (2001) 061601 [hep-th/0105108] [INSPIRE].

[24] E. D'Hoker, J. Estes and M. Gutperle, Exact half-BPS Type IIB interface solutions. I. Local solution and supersymmetric Janus, JHEP 06 (2007) 021 [arXiv:0705.0022] [INSPIRE].

[25] E. D'Hoker, J. Estes and M. Gutperle, Exact half-BPS Type IIB interface solutions. II. Flux solutions and multi-Janus, JHEP 06 (2007) 022 [arXiv:0705.0024] [INSPIRE].

[26] C. Bachas and J. Estes, Spin-2 spectrum of defect theories, JHEP 06 (2011) 005 [arXiv:1103.2800] [INSPIRE].

[27] O. Aharony, L. Berdichevsky, M. Berkooz and I. Shamir, Near-horizon solutions for D3-branes ending on 5-branes, Phys. Rev. D 84 (2011) 126003 [arXiv:1106.1870] [InSPIRE].

[28] B. Assel, C. Bachas, J. Estes and J. Gomis, Holographic duals of $D=3 N=4$ superconformal field theories, JHEP 08 (2011) 087 [arXiv: 1106.4253] [INSPIRE].

[29] B. Assel, C. Bachas, J. Estes and J. Gomis, IIB duals of $D=3 N=4$ circular quivers, JHEP 12 (2012) 044 [arXiv:1210.2590] [INSPIRE].

[30] J. Polchinski and E. Silverstein, Dual purpose landscaping tools: small extra dimensions in AdS/CFT, arXiv:0908.0756.

[31] D. Tsimpis, Supersymmetric AdS vacua and separation of scales, JHEP 08 (2012) 142 [arXiv: 1206.5900] [INSPIRE].

[32] F.F. Gautason, M. Schillo, T. Van Riet and M. Williams, Remarks on scale separation in flux vacua, JHEP 03 (2016) 061 [arXiv:1512.00457] [INSPIRE].

[33] M. Porrati, Higgs phenomenon for $4 D$ gravity in Anti-de Sitter space, JHEP 04 (2002) 058 [hep-th/0112166] [INSPIRE].

[34] M. Porrati, Higgs phenomenon for the graviton in AdS space, Mod. Phys. Lett. A 18 (2003) 1793 [hep-th/0306253] [INSPIRE].

[35] M.J. Duff, J.T. Liu and H. Sati, Complementarity of the Maldacena and Karch-Randall pictures, Phys. Rev. D 69 (2004) 085012 [hep-th/0207003] [InSPIRE].

[36] E. Kiritsis, Product CFTs, gravitational cloning, massive gravitons and the space of gravitational duals, JHEP 11 (2006) 049 [hep-th/0608088] [INSPIRE].

[37] O. Aharony, A.B. Clark and A. Karch, The CFT/AdS correspondence, massive gravitons and a connectivity index conjecture, Phys. Rev. D 74 (2006) 086006 [hep-th/0608089] [INSPIRE].

[38] E. Kiritsis and V. Niarchos, Interacting string multi-verses and holographic instabilities of massive gravity, Nucl. Phys. B 812 (2009) 488 [arXiv:0808.3410] [InSPIRE].

[39] O. Aharony, M. Berkooz and E. Silverstein, Multiple trace operators and nonlocal string theories, JHEP 08 (2001) 006 [hep-th/0105309] [INSPIRE].

[40] O. Aharony, M. Berkooz and B. Katz, Non-local effects of multi-trace deformations in the AdS/CFT correspondence, JHEP 10 (2005) 097 [hep-th/0504177] [INSPIRE].

[41] O. Aharony, G. Gur-Ari and N. Klinghoffer, The holographic dictionary for $\beta$-functions of multi-trace coupling constants, JHEP 05 (2015) 031 [arXiv:1501.06664] [INSPIRE].

[42] O. Aharony et al., Large $N$ field theories, string theory and gravity, Phys. Rept. 323 (2000) 183 [hep-th/9905111] [INSPIRE]. 
[43] A. Karch and L. Randall, Open and closed string interpretation of SUSY CFT's on branes with boundaries, JHEP 06 (2001) 063 [hep-th/0105132] [INSPIRE].

[44] O. DeWolfe, D.Z. Freedman and H. Ooguri, Holography and defect conformal field theories, Phys. Rev. D 66 (2002) 025009 [hep-th/0111135] [INSPIRE].

[45] C. Bachas, J. de Boer, R. Dijkgraaf and H. Ooguri, Permeable conformal walls and holography, JHEP 06 (2002) 027 [hep-th/0111210] [INSPIRE].

[46] J. Erdmenger, Z. Guralnik and I. Kirsch, Four-dimensional superconformal theories with interacting boundaries or defects, Phys. Rev. D 66 (2002) 025020 [hep-th/0203020] [INSPIRE].

[47] O. Aharony, O. DeWolfe, D.Z. Freedman and A. Karch, Defect conformal field theory and locally localized gravity, JHEP 07 (2003) 030 [hep-th/0303249] [INSPIRE].

[48] D. Gaiotto and E. Witten, Janus configurations, Chern-Simons couplings, and the $\theta$-angle in $N=4$ super Yang-Mills theory, JHEP 06 (2010) 097 [arXiv:0804.2907] [INSPIRE].

[49] S. Giombi and I.R. Klebanov, Interpolating between a and F, JHEP 03 (2015) 117 [arXiv: 1409.1937] [INSPIRE].

[50] F.A. Dolan, On superconformal characters and partition functions in three dimensions, $J$. Math. Phys. 51 (2010) 022301 [arXiv:0811.2740] [INSPIRE].

[51] C. Cordova, T.T. Dumitrescu and K. Intriligator, Multiplets of superconformal symmetry in diverse dimensions, arXiv:1612.00809 [INSPIRE].

[52] C. Bachas, M. Bianchi and A. Hanany, $\mathcal{N}=2$ moduli of $A d S_{4}$ vacua: a fine-print study, JHEP 08 (2018) 100 [Erratum ibid. 10 (2018) 032] [arXiv:1711.06722] [INSPIRE].

[53] J. Louis and H. Triendl, Maximally supersymmetric AdS $S_{4}$ vacua in $N=4$ supergravity, JHEP 10 (2014) 007 [arXiv: 1406.3363] [INSPIRE].

[54] C. Cordova, T.T. Dumitrescu and K. Intriligator, Deformations of superconformal theories, JHEP 11 (2016) 135 [arXiv: 1602.01217] [INSPIRE].

[55] O. Lunin, On gravitational description of Wilson lines, JHEP 06 (2006) 026 [hep-th/0604133] [INSPIRE].

[56] J. Gomis and C. Romelsberger, Bubbling defect CFT's, JHEP 08 (2006) 050 [hep-th/0604155] [INSPIRE].

[57] C. Csáki, J. Erlich, T.J. Hollowood and Y. Shirman, Universal aspects of gravity localized on thick branes, Nucl. Phys. B 581 (2000) 309 [hep-th/0001033] [INSPIRE].

[58] A. Miemiec, A power law for the lowest eigenvalue in localized massive gravity, Fortsch. Phys. 49 (2001) 747 [hep-th/0011160] [INSPIRE].

[59] J. Polchinski, String theory. Vol. 2: Superstring theory and beyond, Cambridge University Press, Cambridge U.K. (2006).

[60] R.C. Myers and A. Sinha, Seeing a c-theorem with holography, Phys. Rev. D 82 (2010) 046006 [arXiv: 1006.1263] [INSPIRE].

[61] R.C. Myers and A. Sinha, Holographic c-theorems in arbitrary dimensions, JHEP 01 (2011) 125 [arXiv: 1011.5819] [INSPIRE].

[62] H. Casini, M. Huerta and R.C. Myers, Towards a derivation of holographic entanglement entropy, JHEP 05 (2011) 036 [arXiv:1102.0440] [INSPIRE]. 
[63] H. Casini and M. Huerta, On the RG running of the entanglement entropy of a circle, Phys. Rev. D 85 (2012) 125016 [arXiv:1202.5650] [INSPIRE].

[64] D.L. Jafferis, I.R. Klebanov, S.S. Pufu and B.R. Safdi, Towards the F-theorem: $N=2$ field theories on the three-sphere, JHEP 06 (2011) 102 [arXiv:1103.1181] [INSPIRE].

[65] H. Liu and M. Mezei, A refinement of entanglement entropy and the number of degrees of freedom, JHEP 04 (2013) 162 [arXiv:1202.2070] [INSPIRE].

[66] B. Assel, J. Estes and M. Yamazaki, Large $N$ free energy of $3 d N=4$ SCFTs and $A d S_{4} / C F T_{3}, J H E P 09$ (2012) 074 [arXiv:1206.2920] [INSPIRE].

[67] S. Benvenuti and S. Pasquetti, 3D-partition functions on the sphere: exact evaluation and mirror symmetry, JHEP 05 (2012) 099 [arXiv:1105.2551] [INSPIRE].

[68] T. Nishioka, Y. Tachikawa and M. Yamazaki, $3 D$ partition function as overlap of wavefunctions, JHEP 08 (2011) 003 [arXiv: 1105.4390] [INSPIRE].

[69] T. Damour and I.I. Kogan, Effective Lagrangians and universality classes of nonlinear bigravity, Phys. Rev. D 66 (2002) 104024 [hep-th/0206042] [INSPIRE].

[70] D.Z. Freedman, S.S. Gubser, K. Pilch and N.P. Warner, Renormalization group flows from holography supersymmetry and a $c$ theorem, Adv. Theor. Math. Phys. 3 (1999) 363 [hep-th/9904017] [INSPIRE].

[71] L. Girardello, M. Petrini, M. Porrati and A. Zaffaroni, Novel local CFT and exact results on perturbations of $N=4$ super-Yang-Mills from AdS dynamics, JHEP 12 (1998) 022 [hep-th/9810126] [INSPIRE].

[72] F. Apruzzi et al., Six-dimensional superconformal theories and their compactifications from type IIA supergravity, Phys. Rev. Lett. 115 (2015) 061601 [arXiv:1502.06616] [INSPIRE].

[73] E. D'Hoker, M. Gutperle, A. Karch and C.F. Uhlemann, Warped $A d S_{6} \times S^{2}$ in type IIB supergravity I: local solutions, JHEP 08 (2016) 046 [arXiv: 1606. 01254] [INSPIRE].

[74] E. D'Hoker, M. Gutperle and C.F. Uhlemann, Warped $A d S_{6} \times S^{2}$ in type IIB supergravity II: global solutions and five-brane webs, JHEP 05 (2017) 131 [arXiv:1703.08186] [INSPIRE].

[75] F. Apruzzi, M. Fazzi, A. Passias, D. Rosa and A. Tomasiello, AdS 6 solutions of type-II supergravity, JHEP 11 (2014) 099 [Erratum ibid. 1505 (2015) 012] [arXiv:1406.0852] [INSPIRE].

[76] H. Kim, N. Kim and M. Suh, Supersymmetric AdS $S_{6}$ solutions of type IIB supergravity, Eur. Phys. J. C 75 (2015) 484 [arXiv:1506.05480] [inSPIRE].

[77] D. Gaiotto and J. Maldacena, The gravity duals of $N=2$ superconformal field theories, JHEP 10 (2012) 189 [arXiv: 0904.4466] [INSPIRE].

[78] M. Fazzi, Higher-dimensional field theories from type-II supergravity, arXiv:1712.04447 [INSPIRE].

[79] S. Ferrara, A. Kehagias and D. Lüst, Aspects of weyl supergravity, JHEP 08 (2018) 197 [arXiv: 1806.10016] [INSPIRE]. 